\title{
New perspectives on coping in bipolar disorder
}

\author{
Rodrigo Grassi-Oliveira ${ }^{1}$, Ledo Daruy-Filho ${ }^{1}$, and Elisa Brietzke ${ }^{2}$ \\ 1- Pontifícia Universidade Católica do Rio Grande do Sul, Porto Alegre, RS, Brazil. \\ 2- Universidade Federal de São Paulo, São Paulo, SP, Brazil
}

\begin{abstract}
Bipolar disorder (BD) is a prevalent and highly disabling psychiatric condition. Despite the widely acknowledged importance of psychosocial interventions that involve a complex cognitive, behavioral, and biological process to help patients cope better with their illness, few studies have systematically evaluated coping in BD. Therefore, our objective was to examine recent developments in current research on coping in BD. Several studies have documented a strong association between BD and numerous neuroanatomical and neuropsychological abnormalities, particularly multiple episodes and longer durations of the disorder. The most marked effects of BD encompass brain areas involved in executive function, which may affect the mechanisms underlying an adequate selection of coping strategies. Thus, the ability of individuals to reduce their own stress burden is impaired, increasing vulnerability to stressful life events and negatively affecting the course of BD. Psychosocial interventions that focus on BD should be evaluated for their ability to improve coping abilities, and research on BD should consider neuropsychological impairment and cognitive-behavioral strategies for coping with stress. Keywords: coping skills, bipolar disorder, executive function, stress, adaptation, cognition.
\end{abstract}

Received 6 November; received in revised form 17 December 2010; accepted 18 December 2010. Available on line 28 December 2010

\section{Introduction}

Bipolar disorder (BD) is a prevalent and highly disabling psychiatric condition in which only partially successful treatment is often achieved, even with adequate pharmacotherapy (Perlis et al., 2006). Intense efforts have been directed toward investigating whether psychosocial interventions add to the efficacy of medication in BD (Colom et al., 2008). Most interventions involve a complex cognitive, behavioral, and biological process to help patients adapt to (i.e., avoid or escape) life's demands, which is the generally accepted concept of coping (Compas, 2006). However, the paucity of research focused on systematically evaluating coping in BD is remarkable. Therefore, the aim of the present paper is to briefly present recent developments in research on coping in this disorder.

Rodrigo Grassi-Oliveira and Ledo Daruy-Filho, Developmental Cognitive Neuroscience Research Group, Post-Graduate Program in Psychology, Pontifical Catholic University of Rio Grande do Sul (PUCRS), Brazil. Elisa Brietzke, Program of Intervention in Individuals at Risk Mental States (PRISMA), Department of Psychiatry, Federal University of São Paulo, Brazil. Correspondence regarding this article should be directed to: Rodrigo Grassi-Oliveira, Avenida Ipiranga, 6681, prédio 11, sala 936, Partenon, Porto Alegre, RS, Brazil. CEP 90619-900. Tel: +55 51 3320-3633 ext. 7740. E-mail: rodrigo.grassi@pucrs.br

\section{Basic concepts of coping}

The study of coping as an essential process for understanding how stress affects people's lives is widely accepted by coping researchers. The way people manage stress can both reduce and enhance the effects of stressful life events and adverse conditions with regard to not only emotional distress and short-term functioning, but also long-term functioning and the development of physical and mental health disorders (Skinner, Edge, Altman, \& Sherwood, 2003). Therefore, coping alludes to the cognitive-behavioral strategies used by individuals to adapt to excessive demands that trigger a stress response (Lazarus, 1993). According to Folkman and Lazarus (1980), the concept of coping comprises four essential definitions: ( $i$ ) coping is a process or interaction between the individual and environment, (ii) coping mechanisms likely manage a stressful situation rather than control or overcome it, (iii) the coping process comprises the notion of "evaluation" (i.e., how individuals perceive, interpret, and mentally represent the phenomenon), and (iv) the coping process involves efforts to manage, reduce, or endure internal and external demands that are appraised as exceeding the resources of the person (Antoniazzi, Dell'Aglio, \& Bandeira, 1998).

This model comprises three possible groups of coping strategies: $(i)$ primary control coping (i.e., an attempt to act directly on the source of stress or obtain relief from uncomfortable emotions by engaging 
problem-solving efforts or emotional modulation), (ii) secondary control coping (i.e., an attempt to adjust to the source of stress by engaging acceptance, distancing, cognitive restructuring, or positive reappraisal), and (iii) disengagement coping (i.e., an attempt to escape from the source of stress or emotion by engaging avoidance, denial, or emotional expression; Compas, 2006).

Advances in the methods used for scanning the brain and assessing the structure and function of the central nervous system have provided important tools for the study of neurobiological structures and processes that underlie coping and adaptation to stress. Evidence from the literature suggests that coping may be considered a part of the overall set of executive functions regulated by the prefrontal cortex. Both primary control (e.g., problem solving) and secondary control (e.g., cognitive restructuring, acceptance) engagement coping strategies were related to neuropsychological measures of inhibitory control, whereas disengagement coping (e.g., avoidance) was related to poorer performance on measures of inhibition (Compas, 2006).

Although the concept of coping can be applied to numerous physical, psychological, and psychosocial conditions, a large body of evidence indicates that psychosocial stress might play a major role in the onset and course of BD (Post \& Leverich, 2006). Stressful experiences likely occur in a substantial number of people and have been considered difficult to control, and the choice of coping strategy could be a potential target for psychosocial interventions. In fact, controlled studies that evaluated psychosocial interventions in BD have reported moderate functional improvement, improved social functioning/adaptation, and increased life satisfaction (Miklowitz \& Otto, 2007). Psychosocial interventions for BD patients often include selfmonitoring, the identification of early warning signs of relapse, and techniques for managing adverse conditions and stressful life events (Reinares et al., 2008; Scott, Colom, \& Vieta, 2007; Zaretsky, Lancee, Miller, Harris, $\&$ Parikh, 2008). Although coping may be considered a mechanism of action of these interventions, its role has not yet been rigorously evaluated (Parikh et al., 2007).

\section{Psychophysiological model of stress and coping in bipolar disorder}

According to a recent pathophysiological model of BD (Kapczinski et al., 2008), coping abilities could play a role in modulating the relationship between stress and episode recurrence, which can be directly affected by neurofunctional and neurostructural damage associated with a recurrent course of BD (e.g., prefrontal impairment; Monkul, Malhi, \& Soares, 2005; Robinson et al., 2006). This model assumes that stress and adverse life events are important factors in the onset and course of the disorder (Post, 2007). Subsequently, episode cycling imposes its own rhythm, reducing stress and its role as a trigger of new episodes (Post \& Weiss, 1989). Concerning episode recurrence, although the impact of stressors or adverse life events on the course of the disorder can be attenuated, the effects of stress can be deleterious. Cumulative stress requires continuous efforts of the body to adapt to life's demands.

The term "allostatic load" refers to a cumulative multiple physiological system required for adaptation (McEwen, 1998). Despite the protective characteristics of allostatic adaptation, this repeated resetting of parameters is generally regarded as too high a price to pay if the allostatic process reaches extreme levels or becomes inefficient (McEwen, 2006). The "costs" of allostatic load can be verified through progressive clinical impairment, a high prevalence of medical conditions, mortality, and neurocognitive deficits in BD (Kapczinski et al., 2008; Karlamangla, Singer, McEwen, Rowe, \& Seeman, 2002; Karlamangla, Singer, \& Seeman, 2006; McEwen, 2006; Seeman et al., 2004; Seeman, McEwen, Rowe, \& Singer, 2001). The concept of coping can then be considered a product and modulator of allostatic load.

Our hypothesis considers that coping efforts depend on cognitive function regulated by prefrontal structures and amygdala activation. Several studies on BD have reported impairment in various domains of cognitive function, such as executive function, memory, attention, and processing speed. Severe dysfunctional states were observed in working memory, executive control, concept shifting, fluency, verbal memory, and mental speed (Arts, Jabben, Krabbendam, \& van Os, 2008; Bora, Yucel, \& Pantelis, 2009; Robinson et al., 2006; Torres, Boudreau, \& Yatham, 2007). MartinezAran (2004) detected poorer performance on measures of verbal memory and frontal executive function in BD patients compared with a control group, and low neuropsychological performance was associated with poor functional outcome, including employment status. Verbal memory impairment was related to the duration of the illness and number of previous manic episodes, hospitalizations, and suicide attempts. Other reports indicate the presence of executive deficits despite clinical euthymia (El-Badri, Ashton, Moore, Marsh, \& Ferrier, 2001; Rubinsztein, Michael, Paykel, \& Sahakian, 2000; Zubieta, Huguelet, O’Neil, \& Giordani, 2001).

As the allostatic load increases and mood episodes repeat, the structures involved in executive function, such as the amygdala, prefrontal cortex, and anterior cingulate cortex, undergo anatomical and functional changes. Reduced gray matter volume was demonstrated in the dorsal prefrontal cortex, specifically on the left side (Lopez-Larson, DelBello, Zimmerman, Schwiers, \& Strakowski, 2002; Lyoo et al., 2004). Similar changes were observed in the ventral prefrontal cortex (Almeida et al., 2009), 
particularly in Brodmann's areas 44 and 47 (Frangou, Kington, Raymont, \& Shergill, 2008). Some studies have reported a decreased volume of the left cingulate gyrus, but this is considered an exception (Brambilla et al., 2002). Findings described in the literature regarding amygdala volume in $\mathrm{BD}$ are inconsistent, showing reduced (Berretta, Pantazopoulos, \& Lange, 2007), increased (Altshuler et al., 2005), or unchanged (Almeida et al., 2009) volume in different studies. Much of our current knowledge indicates that some $\mathrm{BD}$ patients have enduring cognitive impairments, which are not dependent on mood, and these deficits might reflect neuropathological changes that occur in specific brain regions implicated in executive function. Multiple episodes and longer durations of the disorder produce a negative and cumulative neuropathological impact (Tham et al., 1997), causing executive function to become progressively less efficient. Similarly, because coping function is directly dependent on the integrity of executive function, such a process also leads to an inefficient use of coping strategies and the consequent adoption of less effective mechanisms in the management of stressful situations. Therefore, because the patient cannot use efficient coping strategies to face adverse life events, the vulnerability to stress increases, together with the development of psychopathology. In summary, BD is characterized by a vicious circle, in which neuropathological and neuropsychological abnormalities produce inefficient or weak coping mechanisms that lead to progressive impairments in coping ability that prevent individuals from successfully managing stressful situations and increase the negative biological and psychological impacts of this condition (Figure 1).

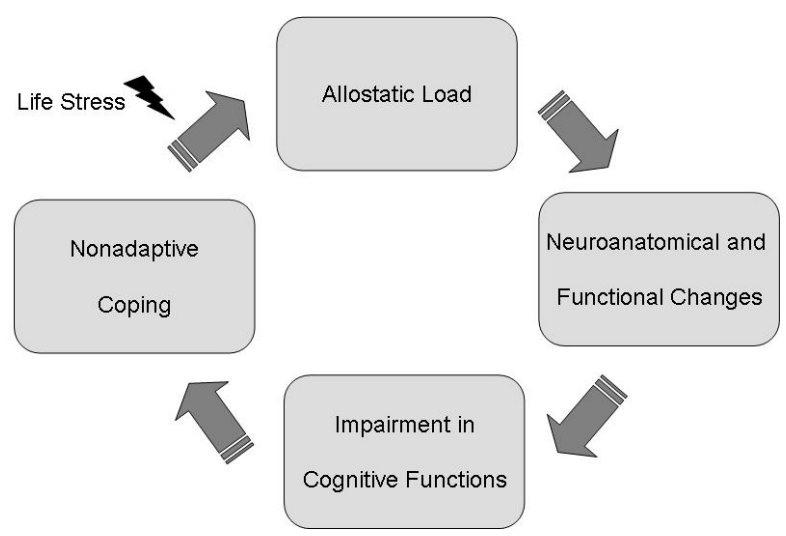

Figure 1. Role of coping in the allostatic load model of BD. Neuroanatomical and neurofunctional changes, including in the prefrontal cortex and amygdala, associated with allostatic load impair several high-order cognitive processes (i.e., executive function). These processes are related to the ability to use adaptive ways of coping. Facing life stress, individuals with coping impairment increase their allostatic load, thus contributing to more neurobiological changes and perpetuating a vicious cycle.

\section{Coping as a possible target for interventions in bipolar disorder}

Despite the limited number of studies that have focused on coping in $\mathrm{BD}$, a few researchers have studied the coping process in the management of its symptoms. The first systematic study was conducted in 1997 (Lam \& Grossman, 1997). Over the past 10 years, research on coping in BD has developed slowly but continuously. Lam and Wong (1997) reported that BD patients' levels of functioning in the areas of work, marital relationships, parenting abilities, and social self-presentations, among others, were highly related to how well they coped with the prodromes of mania. Lam, Wong, and Sham (2001) prospectively studied, over an 18-month period, which types of coping strategies in $\mathrm{BD}$ patients were related to good outcome and a reduction of recurrence and found a relationship between reduced stimulation and prioritizing, reducing the number of tasks to realistic levels and decreasing the chances of experiencing a manic relapse.

The same study reported, however, that a greater number of patients who drank or used other passive strategies in the depression prodromal phase relapsed. One common characteristic of this type of study is that individuals must recognize prodromal symptoms and decide whether they are mild symptoms or false prodromes. They must also recognize whether they are likely to develop into an episode (Lam \& Wong, 2005). When three groups of BD patients (manic, depressed, and in remission) were compared with healthy controls, the manic group had more risk-taking and activecoping responses to depression, which were positively correlated with the severity of mania; however, remitted bipolar patients reported greater rumination (Thomas, Knowles, Tai, \& Bentall, 2007).

Regarding differences between BD subtypes, a recent study demonstrated that subjects with BD types I and II differ in coping mechanisms in response to the prodromes of mania/hypomania. Bipolar disorder type I (BD I) subjects were more likely to seek professional help and reduce stimulation, whereas BD II subjects were more likely to engage in denial and blame. Additionally, BD I subjects tended to use a wider range of coping strategies than BD II subjects. Notably, no association was found between demographic or clinical characteristics and coping strategy, with the exception of the bipolar subtype (Parikh et al., 2007).

Regarding coping with stress, when schizophrenia patients were compared with BD subjects and healthy volunteers, the first group reported lower approach coping than controls, with the BD group reporting intermediate levels. Primary coping strategies, which are typically associated with better outcomes, include actively seeking emotional or instrumental support and guidance, thinking in a logical manner about the stressors and their effects, and using problem-solving skills (Horan et al., 2007). 
Some adaptive approaches to stressful events could result in a positive neuropsychological outcome. Master et al. (2009) studied the use of "emotional approach coping," which is an active effort to acknowledge emotions and explore their meanings to better understand emotional responses surrounding stressors. The use of emotional approach coping was associated with an adaptive stress response, with lower levels of TNF $\alpha$, an immunological stress marker.

Studies on coping in caregivers of BD patients, with coping styles affected by caregiver burden and distress (Chadda, Singh, \& Ganguly, 2007), make this singular population another target for interventions (Bernhard et al., 2006).

Therefore, coping could be seen as a possible target for psychosocial interventions because of the substantial, although partial, causal link between stress and BD (Kapczinski et al., 2008). These interventions should include preventive strategies for population subgroups at high risk of developing BD, therapeutic or pharmacological regimens to minimize the impact of stress on the course of BD (Juruena, Gama, Berk, \& Belmonte-de-Abreu, 2009), and rehabilitation measures for patients in the advanced stages of clinical and cognitive impairment.

\section{Conclusion}

A gap exists between coping theory and the clinical use of coping strategies. The first issue involves the overall assessment of coping abilities, highlighting the need for developing valid and reliable coping instruments. A second issue concerns the lack of integration between the biological bases of $\mathrm{BD}$ and the coping process, considering the body of evidence that links neuropsychological impairment and cognitive-behavioral strategies used for coping with stress. Additionally, coping should be considered a possible mediator of the effects of psychosocial interventions in $\mathrm{BD}$, providing a new basis for evaluating this type of treatment.

\section{References}

Almeida, J.R., Akkal, D., Hassel, S., Travis, M.J., Banihashemi, L., Kerr, N., ...Phillips, M.L. (2009). Reduced gray matter volume in ventral prefrontal cortex but not amygdala in bipolar disorder: significant effects of gender and trait anxiety. Psychiatry Research, 171(1), 54-68.

Altshuler, L., Bookheimer, S., Proenza, M.A., Townsend, J., Sabb, F., Firestine, A.,... Cohen, M.S. (2005). Increased amygdala activation during mania: a functional magnetic resonance imaging study. American Journal of Psychiatry, 162, 1211-1213.

Antoniazzi, A.S., Dell'Aglio, D.D., \& Bandeira, D.R. (1998). O conceito de coping. Estudos de Psicologia, 3, 273-294.

Arts, B., Jabben, N., Krabbendam, L., \& van Os, J. (2008). Metaanalyses of cognitive functioning in euthymic bipolar patients and their first-degree relatives. Psychological Medicine, 38, 771-785.

Bernhard, B., Schaub, A., Kummler, P., Dittmann, S., Severus, E., Seemuller, F.,...Grunze, H. (2006). Impact of cognitive-psychoeducational interventions in bipolar patients and their relatives. European Psychiatry, 21, 81-86.

Berretta, S., Pantazopoulos, H., \& Lange, N. (2007). Neuron numbers and volume of the amygdala in subjects diagnosed with bipolar disorder or schizophrenia. Biological Psychiatry, 62 (8), 884-893.

Bora, E., Yucel, M., \& Pantelis, C. (2009). Cognitive endophenotypes of bipolar disorder: a meta-analysis of neuropsychological deficits in euthymic patients and their first-degree relatives. Journal of Affective Disorders, 113(1-2), 1-20.

Brambilla, P., Nicoletti, M.A., Harenski, K., Sassi, R.B., Mallinger, A.G., Frank, E.,... Soares, J.C. (2002). Anatomical MRI study of subgenual prefrontal cortex in bipolar and unipolar subjects. $\mathrm{Neu}$ ropsychopharmacology, 27, 792-799.

Chadda, R.K., Singh, T.B., \& Ganguly, K.K. (2007). Caregiver burden and coping: a prospective study of relationship between burden and coping in caregivers of patients with schizophrenia and bipolar affective disorder. Social Psychiatry and Psychiatric Epidemiology, 42, 923-930.

Colom, F., Vieta, E., Sanchez-Moreno, J., Goikolea, J.M., Popova, E., Bonnin, C.M., \& Scott, J. (2008). Psychoeducation for bipolar II disorder: an exploratory, 5-year outcome subanalysis. Journal of Affective Disorders, 112(1), 30-35.

Compas, B.E. (2006). Psychobiological processes of stress and coping: implications for resilience in children and adolescents - comments on the papers of Romeo \& McEwen and Fisher et al. Annals of the New York Academy of Sciences, 1094, 226-234.

El-Badri, S.M., Ashton, C.H., Moore, P.B., Marsh, V.R., \& Ferrier, I.N. (2001). Electrophysiological and cognitive function in young euthymic patients with bipolar affective disorder. Bipolar Disorders, 3(2), 79-87.

Folkman, S., \& Lazarus, R.S. (1980). An analysis of coping in a middle-aged community sample. Journal of Health and Social Behavior, 21, 219-239.

Frangou, S., Kington, J., Raymont, V., \& Shergill, S.S. (2008). Examining ventral and dorsal prefrontal function in bipolar disorder: a functional magnetic resonance imaging study. European Psychiatry, 23, 300-308.

Horan, W.P., Ventura, J., Mintz, J., Kopelowicz, A., Wirshing, D., Christian-Herman, J., ...Liberman, R.P. (2007). Stress and coping responses to a natural disaster in people with schizophrenia. Psychiatry Research, 151(1-2), 77-86.

Juruena, M.F., Gama, C.S., Berk, M., \& Belmonte-de-Abreu, P.S. (2009). Improved stress response in bipolar affective disorder with adjunctive spironolactone (mineralocorticoid receptor antagonist): case series. Journal of Psychopharmacology, 23, 985-987.

Kapczinski, F., Vieta, E., Andreazza, A.C., Frey, B.N., Gomes, F.A., Tramontina, J.,...Post, R.M. (2008). Allostatic load in bipolar disorder: implications for pathophysiology and treatment. Neuroscience and Biobehavioral Reviews, 32, 675-692.

Karlamangla, A.S., Singer, B.H., McEwen, B.S., Rowe, J.W., \& Seeman, T.E. (2002). Allostatic load as a predictor of functional decline: MacArthur studies of successful aging. Journal of Clinical Epidemiology, 55, 696-710.

Karlamangla, A.S., Singer, B.H., \& Seeman, T.E. (2006). Reduction in allostatic load in older adults is associated with lower all-cause mortality risk: MacArthur studies of successful aging. Psychosomatic Medicine, 68, 500-507.

Lam, D., \& Wong, G. (1997). Prodromes, coping strategies, insight and social functioning in bipolar affective disorders. Psychological Medicine, 27, 1091-1100.

Lam, D., \& Wong, G. (2005). Prodromes, coping strategies and psychological interventions in bipolar disorders. Clinical Psychology Review, 25, 1028-1042.

Lam, D., Wong, G., \& Sham, P. (2001). Prodromes, coping strategies and course of illness in bipolar affective disorder: a naturalistic study. Psychological Medicine, 31, 1397-1402.

Lam, J.N., \& Grossman, F.K. (1997). Resiliency and adult adaptation in women with and without self-reported histories of childhood sexual abuse. Journal of Traumatic Stress, 10, 175-196.

Lazarus, R.S. (1993). Coping theory and research: past, present, and future. Psychosomatic Medicine, 55, 234-247.

Lopez-Larson, M.P., DelBello, M.P., Zimmerman, M.E., Schwiers, M.L., \& Strakowski, S.M. (2002). Regional prefrontal gray and white matter abnormalities in bipolar disorder. Biological Psychiatry, 52, 93-100.

Lyoo, I.K., Kim, M.J., Stoll, A.L., Demopulos, C.M., Parow, A.M., Dager, S.R.,... Renshaw, P.F. (2004). Frontal lobe gray matter density decreases in bipolar I disorder. Biological Psychiatry, 55, 648-651.

Martinez-Aran, A., Vieta, E., Colom, F., Torrent, C., Sanchez-Moreno, 
J., Reinares, M.,... Salamero, M. (2004). Cognitive impairment in euthymic bipolar patients: implications for clinical and functional outcome. Bipolar Disorders, 6, 224-232.

Master, S.L., Amodio, D.M., Stanton, A.L., Yee, C.M., Hilmert, C.J., \& Taylor, S.E. (2009). Neurobiological correlates of coping through emotional approach. Brain, Behavior, and Immunity, 23(1), 27-35.

McEwen, B.S. (1998). Stress, adaptation, and disease: allostasis and allostatic load. Annals of the New York Academy of Sciences, 840, 33-44.

McEwen, B.S. (2006). Protective and damaging effects of stress mediators: central role of the brain. Dialogues in Clinical Neuroscience, 8, 367-381.

Miklowitz, D.J., \& Otto, M.W. (2007). Psychosocial interventions for bipolar disorder: a review of literature and introduction of the systematic treatment enhancement program. Psychopharmacology Bulletin, 40, 116-131.

Monkul, E.S., Malhi, G.S., \& Soares, J.C. (2005). Anatomical MRI abnormalities in bipolar disorder: do they exist and do they progress? Australian and New Zealand Journal of Psychiatry, 39, 222-226.

Parikh, S.V., Velyvis, V., Yatham, L., Beaulieu, S., Cervantes, P., Macqueen, G.,... Zaretsky, A. (2007). Coping styles in prodromes of bipolar mania. Bipolar Disorders, 9, 589-595.

Perlis, R.H., Ostacher, M.J., Patel, J.K., Marangell, L.B., Zhang, H., Wisniewski, S.R.,... Thase, M.E. (2006). Predictors of recurrence in bipolar disorder: primary outcomes from the Systematic Treatment Enhancement Program for Bipolar Disorder (STEP-BD). American Journal of Psychiatry, 163, 217-224.

Post, R.M. (2007). Kindling and sensitization as models for affective episode recurrence, cyclicity, and tolerance phenomena. Neuroscience and Biobehavioral Reviews, 31, 858-873.

Post, R.M., \& Weiss, S.R. (1989). Sensitization, kindling, and anticonvulsants in mania. Journal of Clinical Psychiatry, 50 (Suppl.), 23-30; discussion 45-27.

Post, R.M., \& Leverich, G.S. (2006). The role of psychosocial stress in the onset and progression of bipolar disorder and its comorbidities: the need for earlier and alternative modes of therapeutic intervention. Development and Psychopathology, 18, 1181-1211.

Reinares, M., Colom, F., Sanchez-Moreno, J., Torrent, C., Martinez-Aran, A., Comes, M.,... Vieta, E. (2008). Impact of caregiver group psychoeducation on the course and outcome of bipolar patients in remission: a randomized controlled trial. Bipolar Disorders, 10, 511-519.
Robinson, L.J., Thompson, J.M., Gallagher, P., Goswami, U., Young, A.H., Ferrier, I.N., \& Moore, P.B. (2006). A meta-analysis of cognitive deficits in euthymic patients with bipolar disorder. Journal of Affective Disorders, 93(1-3), 105-115.

Rubinsztein, J.S., Michael, A., Paykel, E.S., \& Sahakian, B.J. (2000). Cognitive impairment in remission in bipolar affective disorder. Psychological Medicine, 30, 1025-1036.

Scott, J., Colom, F., \& Vieta, E. (2007). A meta-analysis of relapse rates with adjunctive psychological therapies compared to usual psychiatric treatment for bipolar disorders. International Journal of Neuropsychopharmacology, 10(1), 123-129.

Seeman, T.E., Crimmins, E., Huang, M.H., Singer, B., Bucur, A., Gruenewald, T., Berkman, L.F., \& Reuben, D.B. (2004). Cumulative biological risk and socio-economic differences in mortality: MacArthur studies of successful aging. Social Science and Medicine, $58,1985-1997$.

Seeman, T.E., McEwen, B.S., Rowe, J.W., \& Singer, B.H. (2001). Allostatic load as a marker of cumulative biological risk: MacArthur studies of successful aging. Proceedings of the National Academy of Sciences of the United States of America, 98, 4770-4775.

Skinner, E.A., Edge, K., Altman, J., \& Sherwood, H. (2003). Searching for the structure of coping: a review and critique of category systems for classifying ways of coping. Psychological Bulletin, 129, 216-269.

Tham, A., Engelbrektson, K., Mathe, A.A., Johnson, L., Olsson, E., \& Aberg-Wistedt, A. (1997). Impaired neuropsychological performance in euthymic patients with recurring mood disorders. Journal of Clinical Psychiatry, 58(1), 26-29.

Thomas, J., Knowles, R., Tai, S., \& Bentall, R.P. (2007). Response styles to depressed mood in bipolar affective disorder. Journal of Affective Disorders, 100(1-3), 249-252.

Torres, I.J., Boudreau, V.G., \& Yatham, L.N. (2007). Neuropsychological functioning in euthymic bipolar disorder: a meta-analysis. Acta Psychiatrica Scandinavica, 116 (Suppl 434), 17-26.

Zaretsky, A., Lancee, W., Miller, C., Harris, A., \& Parikh, S.V. (2008). Is cognitive-behavioural therapy more effective than psychoeducation in bipolar disorder? Canadian Journal of Psychiatry, 53, 441-448.

Zubieta, J.K., Huguelet, P., O’Neil, R.L., \& Giordani, B.J. (2001). Cognitive function in euthymic bipolar I disorder. Psychiatry Research, 102(1), 9-20. 